\title{
EDITORIAL
}

\section{Glucose tolerance test: varying diagnostic strategies for gestational diabetes mellitus}

\section{Journal of Perinatology (2008) 28, 1-2; doi:10.1038/sj.jp.7211857}

Gestational diabetes mellitus (GDM) is one of the common medical conditions that complicates pregnancy and has been associated with higher incidences of maternal and neonatal complications. ${ }^{1,2}$ It has been demonstrated that treatment of GDM improves perinatal outcomes such that women treated for GDM have lower rates of pre-eclampsia, macrosomia, admissions to the neonatal nursery and composite perinatal complications that include neonatal death, shoulder dystocia, bone fracture and nerve palsy compared to routine prenatal care. ${ }^{3}$ Thus, it is imperative to identify women with GDM at risk of adverse outcomes.

While an oral glucose tolerance test (GTT) is often employed to diagnose GDM, the quantity of the glucose load (75 g vs. $100 \mathrm{~g}$ ) and how frequent the postprandial blood draws $(1,2$ or $3 \mathrm{~h}$ ) should be performed to achieve the optimal diagnostic utility while minimizing the cost and inconvenience remains controversial. In the United States, most clinicians adopt the $100 \mathrm{~g}$ 3-h GTT (checking serum glucose level for fasting, 1-, 2- and 3-h postglucose load) using either the National Diabetes Data Group (NDDG) or the Carpenter-Coustan criteria with two or more abnormal values above the thresholds considered diagnostic. ${ }^{4-6}$ However, the application of the $75 \mathrm{~g} 2$-h GTT to diagnose GDM has also been advocated, ${ }^{7,8}$ while others have explored alternative methods. ${ }^{9,10}$ Since the diagnostic criteria for GDM have not been standardized, how best to diagnose and treat GDM continues to be an area of intense research interest.

In this issue of Journal of Perinatology, Drs Phaloprakarn and Tangitgamol ${ }^{11}$ examined a cohort of women who had $100 \mathrm{~g}$ GTT and developed a new diagnostic scheme for GDM. The authors found that a summation of 1- and 2-h plasma glucose levels post$100 \mathrm{~g}$ GTT using $341 \mathrm{mg}$ per $100 \mathrm{ml}$ and greater as the diagnostic threshold yielded high sensitivity (93.5\%) and specificity (95.2\%) compared to NDDG criteria. By lowering the sensitivity and specificity in changing to this proposed diagnostic test, what is gained or lost? While this novel diagnostic method offers the benefit of patient convenience, less time-consuming, fewer venopunctures and lower costs, one trade-off is that a small percentage of women (6.5\%) who would have been diagnosed with GDM would have been missed and thus not treated for GDM using the proposed diagnostic method. While one could argue that these women may have a milder degree of carbohydrate intolerance and are not necessarily at increased risk for adverse outcomes related to untreated or poorly controlled GDM, others would propose that even women with one elevated $100 \mathrm{~g}$ GTT value are at increased risk for perinatal complications associated with GDM. ${ }^{12,13}$

Additionally, because of the $95 \%$ specificity in the current study as compared to NDDG criteria, $5 \%$ of women who would have not been diagnosed by the NDDG criteria would now be given a diagnosis of GDM. While leading to more falsely diagnosed patients is generally undesirable, in some of these women, such a diagnosis might lead to improved glycemic control and outcomes. Of note in the current study, these 'false-positive' women actually have the greatest mean birthweight and highest rate of macrosomic infants (19\%). However, in others who truly did not have GDM, the consequences of a false diagnosis may be the inconvenience and medical stigma of gestational diabetes.

As Carpenter and Coustan have eloquently stated, the relationship between carbohydrate intolerance and perinatal outcome is likely continuous such that no single diagnostic threshold can truly separate women at high risk of complications from those with no risk at all. ${ }^{14}$ As we seek to refine both the screening and diagnostic tests for GDM, large, multicenter cohort studies, which examine the perinatal outcomes from a variety of testing strategies should be conducted. With the development of continuous glucometers, perhaps an even better diagnostic test may be described using such technology. Further, perhaps simple 'onesize-fits-all' screening or diagnostic tests should be modified within various risk strata of the population in order to improve testing characteristics. It does seem that test characteristics do vary in different populations. ${ }^{15}$ It also may be that by examining not simply diagnostic thresholds, but other mathematical relationships between the test values, we may be better able to identify the population at risk of GDM-related perinatal complications. Thus, the efforts of Drs Phaloprakarn and Tangjitgamol should be applauded, replicated and extended.

The balance between optimal perinatal care and cost-per-GDM diagnosis depends on the disease prevalence in a given population as well as medical resources available to diagnose and treat GDM in order to improve outcomes. With an obstetric population that is increasingly older and overweight, attention to identifying those women at increased risk for perinatal complications associated with 
GDM requires further investigation and probably requires testing strategies with increased sensitivity.

\section{YW Cheng and $\mathrm{AB}$ Caughey \\ Department of Obstetrics, Gynecology and Reproductive Sciences, University of California, San Francisco, CA, USA E-mail:abcmd@berkeley.edu}

\section{References}

1 Hedderson MM, Ferrara A, Sacks DA. Pregnancy hyperglycemia: association with increased risk of spontaneous preterm birth. Am J Obstet Gynecol 2003; 102 $850-856$.

2 Naylor CD, Sermer M, Chen E, Sykora K. Cesarean delivery in relation to birthweight and gestational glucose tolerance: pathophysiology or practice style? Toronto Trihospital Gestational Diabetes Investigators. JAMA 1996; 275: 1165-1170.

3 Crowther CA, Hiller JE, Moss JR, McPhee AJ, Jeffries WS, Robinson JS, for the Australian carbohydrate intolerance study in pregnant women (ACHOIS) trial group. Effect of treatment of gestational diabetes mellitus on pregnancy outcomes. N Engl J Med 2005; 352: $2477-2486$.

4 ACOG Practice Bulletin. Clinical management guidelines for obstetriciangynecologists Number 30, September 2001 (replaces Technical Bulletin Number 200, December 1994) Gestational Diabetes. Obstet Gynecol 2001; 98: 525-538.
5 National Diabetes Data Group. Classification and diagnosis of diabetes mellitus and other categories of glucose intolerance. Diabetes 1979; 28: 1039-1057.

6 Carpenter MW, Coustan DR. Criteria for screening tests for gestational diabetes. Am J Obstet Gynecol 1982; 144: 768-773.

7 American Diabetes Association position statement. Standards of medical care in diabetes. Diabetes Care 2006; 28: S4-\$42

8 World Health Organization. Prevention of Diabetes Mellitus. WHO: Geneva, 1994

9 Atilano LC, Lee-Parritz A, Lieberman E, Cohen AP, Barbieri RL. Alternative methods of diagnosing gestational diabetes mellitus. Am J Obstet Gynecol 1999; 181: 1158-1161.

10 Jakobi P, Solt I, Weissman A. A $2 \mathrm{~h}$ versus the $3 \mathrm{~h} 100 \mathrm{~g}$ glucose tolerance test for diagnosing gestational diabetes mellitus. J Perinat Med 2004; 32: 320-322.

11 Phaloprakarn C, Tangitgamol S. Diagnosis of gestational diabetes mellitus using a modified 100-gram oral glucose tolerance test. J Perinatol 2007 (in press)

12 Stamilio DM, Olsen T, Ratcliffe S, Sehdev HM, Macones GA. False-positive 1-hour glucose challenge test and adverse perinatal outcomes. Obstet Gynecol 2004; 103 $148-156$.

13 McLaughlin GB, Cheng YW, Caughey AB. Women with one elevated 3-hour glucose tolerance test value: are they at risk for adverse perinatal outcomes? Am J Obstet Gynecol 2006; 194: 316-319.

14 Coustan DR, Carpenter MW. The diagnosis of gestational diabetes. Diabetes Care 1998; 21(Suppl 2): B5-B8.

15 Esakoff TF, Cheng YW, Caughey AB. What is the optimal threshold to screen for gestational diabetes among different ethnicities? Am J Obstet Gynecol 2005; 193 $1040-1044$ 